\title{
Super-resolution of Satellite Imagery Using a Wavelet Multiscale-based Deep Convolutional Neural Network Model
}

\author{
Nour Aburaed $^{\mathrm{a}}$, Alavikunhu Panthakkan ${ }^{\mathrm{a}}$, Mina Al-Saad ${ }^{\mathrm{a}}$, Marwa Chendeb El Rai ${ }^{\mathrm{a}}$, Saeed Al \\ Mansoori $^{\mathrm{b}}$, Hussain Al Ahmad ${ }^{\mathrm{a}}$, and Stephen Marshall ${ }^{\mathrm{c}}$ \\ ${ }^{a}$ University of Dubai, Dubai, UAE \\ ${ }^{\text {b} M o h a m m e d ~ B i n ~ R a s h i d ~ S p a c e ~ C e n t r e, ~ D u b a i, ~ U A E ~}$ \\ ${ }^{\mathrm{c}}$ University of Strathclyde, Glasgow, UK
}

\begin{abstract}
Nowadays, satellite images are used in various governmental applications, such as urbanization and monitoring the environment. Spatial resolution is an element of crucial impact on the usage of remote sensing imagery. As such, increasing the spatial resolution of an image is an important pre-processing step that can improve the performance of various image processing tasks, such as segmentation. Once a satellite is launched, the more practical solution to improve the resolution of its captured images is to use Single Image Super Resolution (SISR) techniques. In the recent years, Deep Convolutional Neural Networks (DCNNs) have been recognized as a highly effective tool to reconstruct a High Resolution (HR) image from its Low Resolution (LR) counterpart, which is an open problem due to the inherent difficulty of estimating the missing high frequency components. The aim of this research paper is to design and implement a satellite image SISR algorithm by estimating high frequency details through training Deep Convolutional Neural Network (DCNNs) with respect to wavelet analysis. The goal is to improve the spatial resolution of multispectral remote sensing images captured by DubaiSat-2 satellite. The accuracy of the proposed algorithm is assessed using several metrics such as Peak Signal-to-Noise Ratio (PSNR), Wavelet-based Signal-to-Noise Ratio (WSNR) and Structural Similarity Index Measurement (SSIM).
\end{abstract}

Keywords: Remote Sensing, Wavelet Transform, Super Resolution, Convolutional Neural Network, Deep Learning

\section{INTRODUCTION}

Remote sensing imagery has been a trending topic ever since the 70s when the first Landsat image was captured. The variety of satellites that emerged since then facilitate different remote sensing applications, such as urbanization, Land Cover Land Use (LCLU), oceanography, and vegetation. Most of these applications require high spatial resolution in order to achieve satisfactory results with high accuracy. Once a satellite is launched, the resolution of the captured image can no longer be controlled by hardware means. Additionally, there exists a trade off between the cost and the resolution of the image. Therefore, the most practical solution is to estimate the High Resolution (HR) image from the existing Low Resolution (LR) one. This process is known as Single Image Super Resolution (SISR). SISR is an ill-posed problem due to the difficulty of estimating the missing high frequency details from the LR image. One of the traditional solutions in literature include interpolation, such as bilinear, ${ }^{1}$ bicubic, ${ }^{2}$ and lanczos. ${ }^{3}$ This technique is used in real-time applications due to its low computational complexity. However, interpolation techniques cannot estimate the high frequency details due to their low-pass filtering process. Thus, the results often appear blurry with artifacts around the edges. A more successful alternative to interpolation is example-based methods. ${ }^{4}$ These methods include machine learning algorithms, such as Neighbour Embedding ${ }^{578}$, Sparse Coding ${ }^{91011}$, Regression Trees ${ }^{121314}$, and Deep Learning (DL). DL is a sub-field of machine learning, which is in turn a sub-field of Artificial Intelligence. DL is the process of making Deep Neural Networks (DNNs) capable of supervised learning using labeled data. Convolutional Neural

Further author information: (Send correspondence to N.A.)

N.A.: E-mail: noaburaed@ud.ac.ae

A.P.: E-mail: apanthakkan@ud.ac.ae 
Networks (CNNs) are a special type of Neural Networks that are capable of performing various image processing tasks depending on their architecture as well as the formats of their inputs and outputs. When a CNN has several hidden layers, it is known as Deep CNN (DCNN). DCNNs have proven superiority in SISR ${ }^{1516171819}$, and they are currently being widely utilized for this purpose. One of the most well-known CNNs for such task and the one of interest in this paper is Super-Resolution CNN (SRCNN) ${ }^{20} \cdot{ }^{21}$ In this paper, wavelet decomposition tasks are introduced alongside SRCNN to enhance LR image in the YCBCR color domain. In the paper authored by Aburaed et al., ${ }^{22}$ it has been hypothesized that if the luminance channel is processed with a DCNN by itself, then the result is similar to the one obtained by processing RGB channels using the same DCNN. The same hypothesis is used here, while processing the luminance channel in the wavelet domain instead of the spatial domain to enhance the results further. This modified SRCNN will be referred to as Wavelet SRCNN (WSRCNN). DubaiSat- $2^{23}$ images are used for the purpose of training, testing, and evaluating WSRCNN.

The rest of the paper is organized as follows: Section 2 describes the dataset used in this study. Section 3 explains the methodology used, including SRCNN, wavelet decomposition, and the training strategy for WSRCNN. Section 4 discusses the results obtained from the training. Finally, Section 5 draws the conclusions and the future direction of this study.

\section{DATASET}

A set of 1000 images of size $512 \times 512$ were collected from DubaiSat-2. These images are used as ground truth images. This set was downsampled to size $256 \times 256$ in order to be used to train the WSRCNN. Similarly, in order to test the network, 200 images of size $512 \times 512$ are used as ground truth, and they were downsampled to size $256 \times 256$, which were used to evaluate the network. The dataset maintains variety of structures to ensure consistency and to avoid learning bias and overfitting. The images contain a balanced amounts of desert areas, water bodies, buildings, vegetation, and roads.

\section{METHODOLOGY}

The following subsections explain the different components of the proposed SISR algorithm and the training strategy for WSRCNN.

\subsection{Super-Resolution Convolutional Neural Network (SRCNN)}

SRCNN is a feed-forward CNN that consists of three main parts; patch-extraction, non-linear mapping, and reconstruction. Due to its simplicity, it is faster than other SISR algorithms and has superior performance in most cases in terms of Peak Signal-to-Noise Ratio (PSNR). It is necessary to upscale the low-resolution image to the same size as the output HR images by applying bicubic interpolation before feeding them to the network. In the patch extraction layer, the overlapping patches are extracted from the low resolution interpolated image and then each extracted patch is represented as a high dimensional vector and this can be expressed as Equation (1): ${ }^{24}$

$$
F_{1}(Y)=\max \left(0, W_{1} * Y+B_{1}\right)
$$

where $Y, W_{1}$, and $B_{1}$ are the low-resolution image, filter, and bias, respectively. $W_{1}$ is $c \times f_{1} \times f_{1} \times n_{1}$, where $c$ corresponds to the number of channels in the input image, $f_{1}$ is the filter spatial size, and $n_{1}$ is the number of convolution kernels. The purpose of the second convolutional layer is to perform non-linear mapping of the extracted low dimensional vector features to high dimensional vector features, this step can be expressed as Equation (2):

$$
F_{2}(Y)=\max \left(0, W_{2} * F_{1}(Y)+B_{2}\right)
$$

where $W_{2}$ is $n_{2} \times f_{2} \times f_{2} \times n_{1}$, and $B_{2}$ is the bias. After the non-linear mapping, image reconstruction is used to generate the final HR image from HR features as given in Equation (3):

$$
F(Y)=\left(W_{3} * F_{2}(Y)+B_{3}\right)
$$

where $W_{3}$ is the kernel of the reconstruction convolution layer with size of $n_{2} \times f_{3} \times f_{3}$, and $B_{3}$ is the bias. 


\subsection{Multiscale Wavelet Decomposition}

Wavelet analysis is a successor of Fourier analysis and is based on the same principle. An image can be translated to the frequency domain by choosing a set of linearly dependent functions, and the image is represented as a sum of these functions with coefficients. Thus, the changes of the image can be tracked in the frequency spectrum. Also, this allows the transition to multi-scale representation of the image. Wavelet image representation is more compact than the spatial representation. Additionally, the spatial image can be estimated by using a fraction of the wavelet coefficients. Hence, wavelet transformation is widely used in image compression and scaling. Discrete Wavelet Decomposition (DWT) ${ }^{25}$ is performed by applying two filters sequentially; low-pass and high-pass. Since an image is a 2D signal, the filters are applied both horizontally and vertically. The result is four sub-bands that are half the width and height of the original image. The first sub-band is a smaller copy of the image (LL), and the remaining sub-bands, horizontal $(\mathrm{HL})$, vertical $(\mathrm{LH})$, and diagonal $(\mathrm{HH})$, contain information about the details of the image. Figure 1 shows an example of DWT.
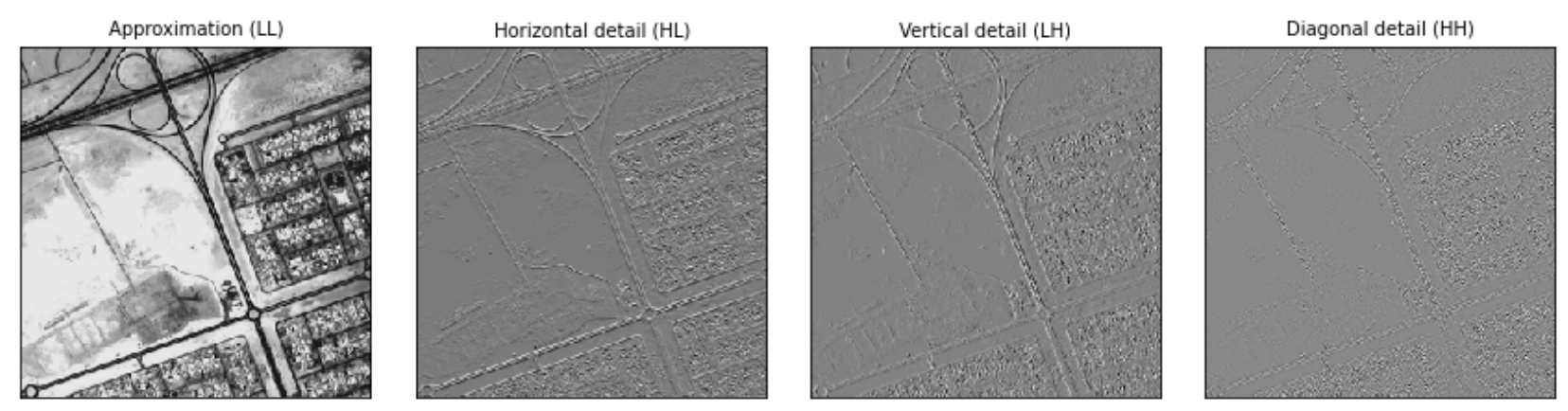

Figure 1: Wavelet decomposition.

\subsection{Training Strategy}

The first stage of training WSRCNN is transforming the image from RGB to YCBCR domain. This color domain consists of three channels, Luminance (Y), Chroma Blue (CB), and Chroma Red (CR). The human eye is more sensitive to the $\mathrm{Y}$ component, while it is less sensitive to $\mathrm{CB}$ and $\mathrm{CR}$ components. Therefore, the $\mathrm{Y}$ channel needs to be accurate, but CB and CR need not to be accurate. Hence, $\mathrm{Y}$ is processed using SRCNN, but CB and $\mathrm{CR}$ are processed using traditional interpolation methods. In this study, bicubic interpolation is used for this purpose. This is done to reduce the processing time and to ensure that the weights in the network are focused on the components that affect the overall visual quality. Before processing Y with SRCNN, it is transformed to the wavelet domain, which gives LL, LH, HL, and HH components. Each component is trained with SRCNN separately. Since SRCNN has fast performance, processing each component individually does not compromise the computational cost. This allows the network to observe the changes on frequency level in the Y channel, which boosts the overall quality. The result of each SRCNN are combined and transformed back to the spatial domain using Inverse DWT (IDWT). Afterward, the result of enhancing Y, CB, and CR are combined together and transformed back to the RGB color domain in order to obtain the final HR image. A flowchart of WSRCNN is shown in Figure 2.

\section{RESULTS AND DISCUSSION}

The results of WSRCNN have been evaluated in terms of PSNR, Structural Similarity Index Measurement (SSIM), and Wavelet-based Signal-to-Noise Ratio (WSNR). Table 1 summarizes the results of 9 random test images. The performance is compared against bicubic interpolation, which WSRCNN shows superiority. The performance of WSRCNN is also compared against using RGB images as opposed to Y channel by itself. The latter shows either similar or better performance than RGB. Figure 3 shows visual comparisons between WSRCNN and bicubic interpolation against the ground truth images. The images shown are cropped in this paper due to space limitation and for better observation and demonstration. It is observed that WSRCNN is visually 


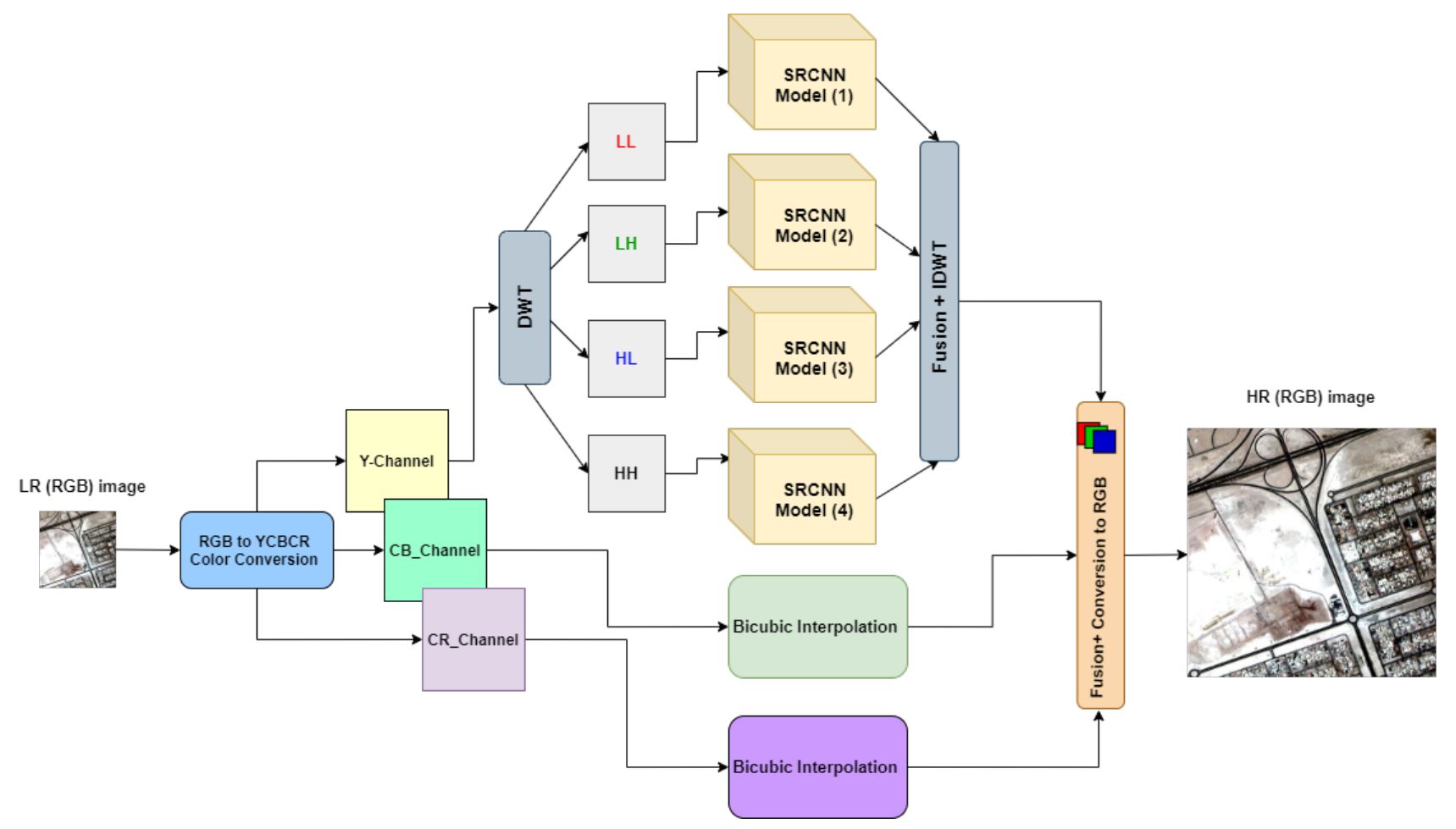

Figure 2: A flowchart of the training strategy for WSRCNN

more similar to the ground truth image, while bicubic interpolation results show blurriness and over-smoothness around the edges and image details.

\section{CONCLUSION}

In this paper, a wavelet approach to training SRCNN in the wavelet domain is introduced as WSRCNN. The image is first converted to YCBCR color domain, and then wavelet transformation is applied to the Y channel only. Each of the four wavelet sub-bands is processed with SRCNN separately. This allows direct enhancement to high and low frequency components, which makes the resultant image retain its details without over-smoothing. The enhanced Y channel is fused with interpolated CB and CR to get the final HR image. This approach shows superiority in terms of PSNR, SSIM, and WSNR. Visual inspection shows that the HR image obtained from WSRCNN is visually similar to the ground truth image. Additionally, it is less computationally extensive as opposed to training the model using RGB.

\section{REFERENCES}

[1] William H. Press, Saul A. Teukolsky, W. T. V. B. P. F. in [Numerical recipes in C: the art of scientific computing (2nd ed.)], 123-128, Cambridge University Press, New York (1992).

[2] Keys, R., "Cubic convolution interpolation for digital image processing," IEEE Transactions on Acoustics, Speech, and Signal Processing 29(6), 1153-1160 (1981).

[3] Turkowski, K., "Filters for common resampling tasks," Graphics gems , 147-165 (1990).

[4] Salvador, J. in [Example-Based Super Resolution], 162, Academic Press (2016).

[5] Yang, S., Wang, Z., Zhang, L., and Wang, M., "Dual-geometric neighbor embedding for image super resolution with sparse tensor," IEEE Transactions on Image Processing 23(7), 2793-2803 (2014).

[6] Song, S., Li, Y., Liu, J., and Quo, Z., "Joint sub-band based neighbor embedding for image super-resolution," in [2016 IEEE International Conference on Acoustics, Speech and Signal Processing (ICASSP)], 1661-1665 (2016). 


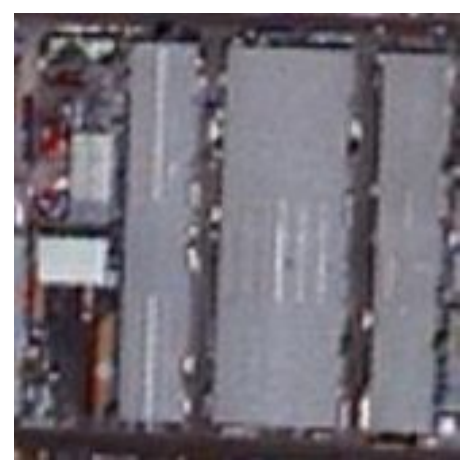

(a) Sat1- bicubic interpolation

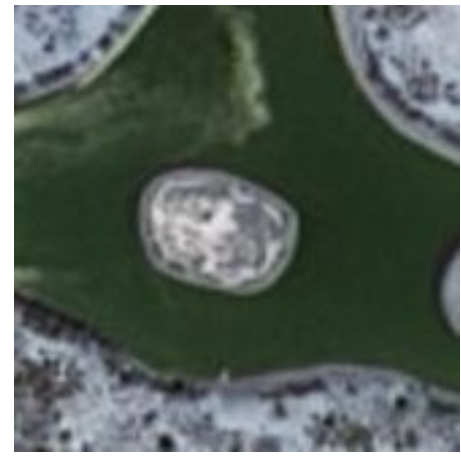

(d) Sat2- bicubic interpolation

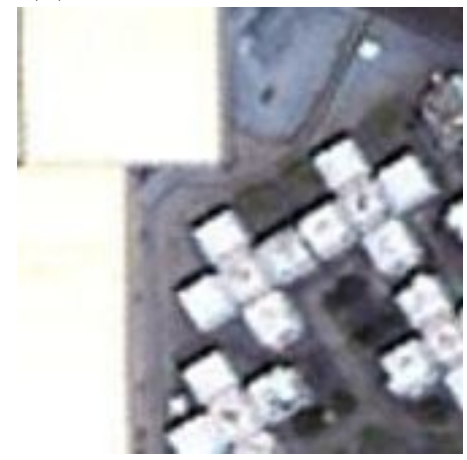

(g) Sat3- bicubic interpolation

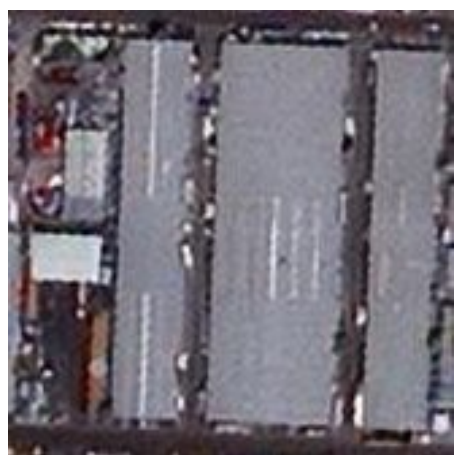

(b) Sat1- WSRCNN

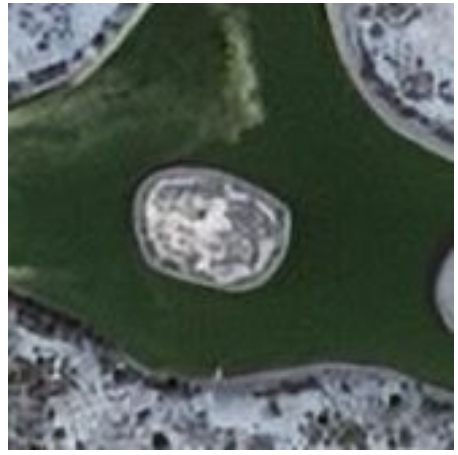

(e) Sat2- WSRCNN

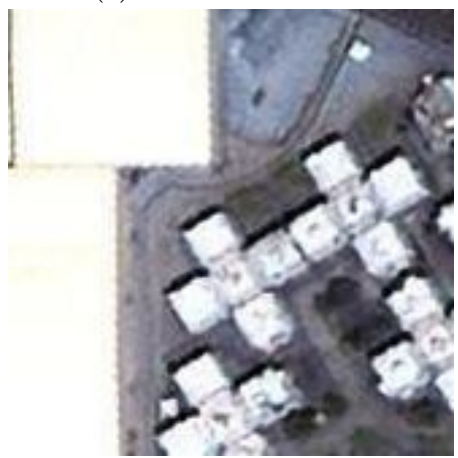

(h) Sat3- WSRCNN

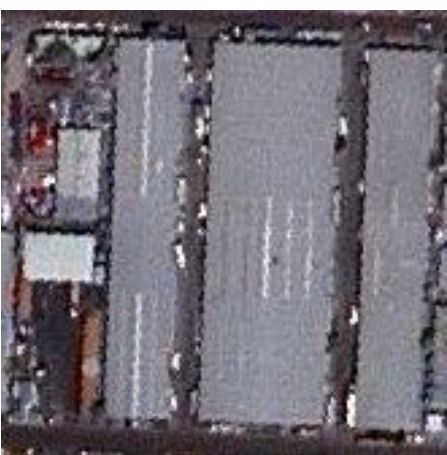

(c) Sat1- ground truth

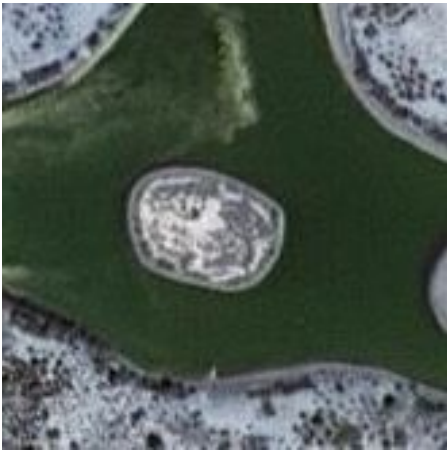

(f) Sat2- ground truth

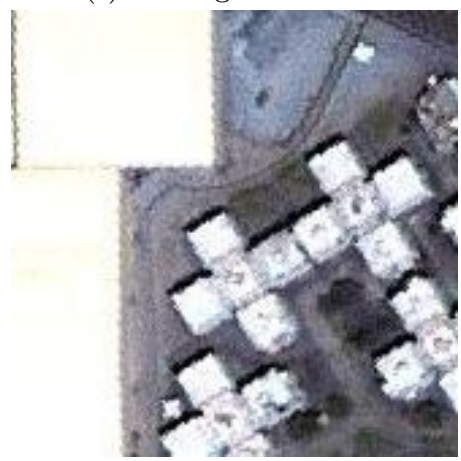

(i) Sat3- ground truth

Figure 3: Comparison of visual quality between bicubic interpolation and WSRCNN against ground truth images.

[7] Alonso-Fernandez, F., Farrugia, R. A., and Bigun, J., "Iris super-resolution using iterative neighbor embedding," in [2017 IEEE Conference on Computer Vision and Pattern Recognition Workshops (CVPRW)], $655-663$ (2017).

[8] Jiang, J., Ma, X., Chen, C., Lu, T., Wang, Z., and Ma, J., "Single image super-resolution via locally regularized anchored neighborhood regression and nonlocal means," IEEE Transactions on Multimedia 19(1), $15-26$ (2017).

[9] Zhu, X., Liu, L., Wang, X., and Wang, J., "Super-resolution reconstruction via multiple sparse dictionary combined with sparse coding," in [2016 IEEE International Conference on Information and Automation (ICIA)], 1720-1725 (2016).

[10] Gao, X. and Xiong, H., "A hybrid wavelet convolution network with sparse-coding for image superresolution," in [2016 IEEE International Conference on Image Processing (ICIP)], 1439-1443 (2016).

[11] Gu, P. and Zheng, L., "Fast low-dose computed tomography image super-resolution reconstruction via sparse 
Table 1: Results summary of WSRCNN and performance comparison against bicubic interpolation and using RGB channels.

\begin{tabular}{|c|c|c|c|c|c|c|}
\hline \multirow{2}{*}{ Image } & \multicolumn{3}{|c|}{ Bicubic Interpolation } & \multicolumn{3}{c|}{ Wavelet SRCNN } \\
\cline { 2 - 7 } & SSIM & PSNR & WSNR & SSIM & PSNR & WSNR \\
\hline Sat1-YCBCR & 0.999985 & 70.9756 & 88.3121 & $\mathbf{0 . 9 9 9 9 9 7}$ & $\mathbf{7 3 . 0 1 4 3}$ & $\mathbf{9 3 . 2 6 2 2}$ \\
\hline Sat1-RGB & 0.999985 & 90.9735 & 88.3271 & 0.999997 & 72.8941 & 92.8623 \\
\hline Sat2-YCBCR & 0.999982 & 70.8262 & 88.2032 & $\mathbf{0 . 9 9 9 9 9 7}$ & $\mathbf{7 3 . 1 5 9 6}$ & $\mathbf{9 3 . 8 8 3 2}$ \\
\hline Sat2-RGB & 0.999982 & 70.8254 & 88.2167 & 0.999997 & 73.0298 & 93.1229 \\
\hline Sat3-YCBCR & 0.999984 & 70.8317 & 80.207173 & $\mathbf{0 . 9 9 9 9 9 7}$ & $\mathbf{7 2 . 8 0 9 2 0 8}$ & $\mathbf{9 3 . 2 3 1 4}$ \\
\hline Sat3-RGB & 0.999984 & 70.8316 & 88.2242 & 0.999997 & 72.6919 & 92.6259 \\
\hline Sat4-YCBCR & 0.999989 & 70.0860 & 90.364903 & $\mathbf{0 . 9 9 9 9 9 8}$ & $\mathbf{7 3 . 6 3 1 7 3 7}$ & $\mathbf{9 4 . 3 6 6 0}$ \\
\hline Sat4-RGB & 0.999989 & 72.0840 & 90.3910 & 0.999997 & 73.5253 & 93.3883 \\
\hline Sat5-YCBCR & 0.999990 & 73.5966 & 90.5664 & $\mathbf{0 . 9 9 9 9 9 8}$ & $\mathbf{7 5 . 7 3 1 8}$ & $\mathbf{9 5 . 7 7 3 3}$ \\
\hline Sat5-RGB & 0.999990 & 73.5953 & 90.5844 & 0.999998 & 75.6156 & 95.1051 \\
\hline Sat6-YCBCR & 0.999993 & 74.7250 & 91.5728 & $\mathbf{0 . 9 9 9 9 9 9}$ & $\mathbf{7 6 . 8 4 7 6}$ & $\mathbf{9 6 . 9 5 1 6}$ \\
\hline Sat6-RGB & 0.999993 & 74.7235 & 91.6097 & 0.999999 & 76.7248 & 96.1230 \\
\hline Sat7-YCBCR & 0.999995 & 76.1407 & 92.9929 & $\mathbf{0 . 9 9 9 9 9 9}$ & $\mathbf{7 8 . 2 9 4 4}$ & $\mathbf{9 8 . 0 6 0 9}$ \\
\hline Sat7-RGB & 0.999995 & 76.1447 & 93.0126 & 0.999999 & 78.2272 & 97.5249 \\
\hline Sat8-YCBCR & 0.999993 & 74.8735 & 92.0436 & $\mathbf{0 . 9 9 9 9 9 9}$ & $\mathbf{7 6 . 7 2 5 7}$ & $\mathbf{9 6 . 6 1 0 0}$ \\
\hline Sat8-RGB & 0.999993 & 74.8747 & 92.0636 & 0.999999 & 76.6326 & 96.1235 \\
\hline Sat9-YCBCR & 0.999987 & 72.8011 & 89.4624 & $\mathbf{0 . 9 9 9 9 9 8}$ & $\mathbf{7 4 . 8 8 3 7}$ & $\mathbf{9 5 . 3 7 6 1}$ \\
\hline Sat9-RGB & 0.999987 & 72.7993 & 89.4900 & 0.999996 & 74.6377 & 92.6700 \\
\hline
\end{tabular}

coding and random forests," in [2019 IEEE 8th Joint International Information Technology and Artificial Intelligence Conference (ITAIC)], 1400-1403 (2019).

[12] Liu, Z., Siu, W., and Huang, J., "Image super-resolution via weighted random forest," in [2017 IEEE International Conference on Industrial Technology (ICIT)], 1019-1023 (2017).

[13] Huang, J. and Siu, W., "Learning hierarchical decision trees for single-image super-resolution," IEEE Transactions on Circuits and Systems for Video Technology 27(5), 937-950 (2017).

[14] Deng, L., Hu, J., and Qi, J., "Super-resolution reconstruction of finger vein image based on regression tree model," in [2020 Chinese Control And Decision Conference (CCDC)], 3796-3801 (2020).

[15] Tom, F., Sharma, H., Mundhra, D., Dastidar, T. R., and Sheet, D., "Learning a deep convolution network with turing test adversaries for microscopy image super resolution," in [2019 IEEE 16th International Symposium on Biomedical Imaging (ISBI 2019)], 1391-1394 (2019).

[16] Chen, C. and Qi, F., "Single image super-resolution using deep cnn with dense skip connections and inception-resnet," in [2018 9th International Conference on Information Technology in Medicine and Education (ITME)], 999-1003 (2018).

[17] Brown, K. and Hoyt, K., "Deep learning in spatiotemporal filtering for super-resolution ultrasound imaging," in [2019 IEEE International Ultrasonics Symposium (IUS)], 1114-1117 (2019).

[18] Jiang, X., Xu, Y., Wei, P., and Zhou, Z., "Ct image super resolution based on improved srgan," in [2020 5th International Conference on Computer and Communication Systems (ICCCS)], 363-367 (2020).

[19] Wang, Z., Chen, J., and Hoi, S. C. H., "Deep learning for image super-resolution: A survey," CoRR abs/1902.06068 (2019).

[20] Dong, C., Loy, C. C., He, K., and Tang, X., "Learning a deep convolutional network for image superresolution," in [Computer Vision - ECCV 2014], Fleet, D., Pajdla, T., Schiele, B., and Tuytelaars, T., eds., 184-199, Springer International Publishing, Cham (2014).

[21] Dong, C., Loy, C. C., He, K., and Tang, X., "Image super-resolution using deep convolutional networks," IEEE Transactions on Pattern Analysis and Machine Intelligence 38(2), 295-307 (2016). 
[22] Aburaed, N., Panthakkan, A., III, S. A., and Al-Ahmad, H., "Super resolution of DS-2 satellite imagery using deep convolutional neural network," in [Image and Signal Processing for Remote Sensing XXV], Bruzzone, L. and Bovolo, F., eds., 11155, 485 - 491, International Society for Optics and Photonics, SPIE (2019).

[23] Bushahab, A., Al-Mansoori, S., Al-Suwaidi, K., Matroushi, H. A., Al-Tunaiji, E., and Shamsi, M. A., "Cal/Val activities for DubaiSat-2 performance assessment," in [Sensors, Systems, and Next-Generation Satellites XVIII], Meynart, R., Neeck, S. P., and Shimoda, H., eds., 9241, 319 - 327, International Society for Optics and Photonics, SPIE (2014).

[24] Dong, C., Loy, C., He, K., and Tang, X., "Image super-resolution using deep convolutional networks," in [2016 IEEE Transactions on Pattern Analysis and Machine Intelligence], 28, 295-307 (2016).

[25] Moulin, P., "Chapter 6 - multiscale image decompositions and wavelets," in [The Essential Guide to Image Processing], Bovik, A., ed., 123 - 142, Academic Press, Boston (2009). 